\title{
INTERSECTIONS OF TOTALLY REAL AND HOLOMORPHIC DISKS
}

\author{
TOM DUChamp and Franc Forstnerič
}

\begin{abstract}
It is shown that a holomorphically embedded open disk in $\mathbb{C}^{2}$ and a totally real embedded open disk which have a common smooth boundary have nontrivial intersection.
\end{abstract}

\section{Introduction}

It is now clear, through the work of Gromov and others, that there is a strong relationship between the theory of Lagrangian immersions into symplectic manifolds and the theory of totally real immersions into complex manifolds. The relation follows from the fact that the Grassmann space of Lagrangian subspaces of $\mathbb{R}^{2 n}$ is homotopy equivalent to the Grassmann space of totally real subspaces of $\mathbb{C}^{n}$.

There are surprising differences between the two theories, however. Recall that complex 2-space, $\mathbb{C}^{2}$, is a symplectic manifold with symplectic form

$$
\omega=\frac{i}{2}\left(d z^{1} \wedge d \bar{z}^{1}+d z^{2} \wedge d \bar{z}^{2}\right) .
$$

Let $\psi: \Delta \rightarrow \mathbb{C}^{2}$ be a symplectic embedding of the open unit disk in $\mathbb{C}$, that is, suppose that the pull-back $\psi^{*} \omega$ is a symplectic form on $\Delta$. It is not difficult to prove that every holomorphic embedding of $\Delta$ is symplectic. Assume further that $\psi$ extends to a smooth embedding of the closed disk $\bar{\Delta}$. There is no Lagrangian embedding of $\Delta$ whose boundary coincides with $\psi(\partial \Delta)$. For suppose that $\phi$ is such an embedding. Then $\psi(\bar{\Delta})$ and $\phi(\bar{\Delta})$ are homologous relative to the boundary; hence,

$$
\int_{\Delta} \phi^{*} \omega=\int_{\Delta} \psi^{*} \omega
$$

The second author was partially supported by the Research Council of the Republic of Slovenia. 
which is a contraction because the first integral is 0 and the second is positive.

On the other hand, it is casy to construct examples of a pair consisting of a holomorphically embedded disk and a totally real disk which have a. common smooth boundary (see Section 4 below for one such example). In this paper the intersection theory of such a pair is investigated. The main result is the following.

Intersection Theorem. If a holomorphically embedded disk and a totally real embedded disk in $\mathbb{C}^{2}$ have a common boundary then they intersect in at least one interior point. More precisely, let $\psi: \bar{\Delta} \rightarrow \mathbb{C}^{2}$ be a smooth embedding of the closed unit disk, holomorphic on $\Delta ;$ and let $\phi: \bar{\Delta} \rightarrow \mathbb{C}^{2}$ be a smooth totally real embedding such that

$$
\psi(\partial \Delta)=\phi(\partial \Delta)
$$

Then the intersection $\psi(\Delta) \cap \phi(\Delta)$ is non-empty.

The proof of the theorem is by contradiction: Suppose the theorem is false. Then there are embeddings $\psi$ and $\phi$ of $\bar{\Delta}$ with $\psi(\Delta) \cap \phi(\Delta)=\emptyset$ and $\psi(\partial \Delta)=\phi(\partial \Delta)$. We will show how to deform the images $\psi(\bar{\Delta})$ and $\phi(\bar{\Delta})$ to produce two totally real embeddings which abut smoothly along the boundary $\psi(\partial \Delta)$ in such a way that the union of the images of the deformed totally real embeddings defines a totally real embedding of the two sphere into $\mathbb{C}^{2}$. Bishop [1] (see also Wells [5]) proved that there is no totally real embedding of the two sphere into $\mathbb{C}^{2}$.

Acknowledgment. We wish to thank Professor E. L. Stout for bringing this problem to our attention.

\section{Properties of totally real embeddings}

Before beginning the proof it is necessary to review several conditions which are equivalent to the total reality condition and to define an index for totally real embeddings of annuli.

2.1. Conditions for total reality. An embedding $\psi: U \rightarrow \mathbb{C}^{2}$, $U \subset \mathbb{C}$ a region, is said to be totally real if for all $\zeta \in U$ the intersection $\psi_{*} T_{\zeta} U \cap J \psi_{*} T_{\zeta} U$ is trivial. (Here $T U$ denotes the real tangent bundle of $U$ and $J$ the complex structure tensor of $\mathbb{C}^{2}$.) Let $(z, w)$ denote complex coordinates on $\mathbb{C}^{2}$ and $(u, v)$ arbitrary real coordinates on $U$. The next lemma gives several characterizations of the total reality condition. They are more or less well-known and easily verified, so we leave it to the reader to check them. (See Stout-Zame [4] for a discussion of total reality). 
Lemma 1. Let $\psi: U \rightarrow \mathbb{C}^{2}$ be on embedding, $U \subset \mathbb{C}$, defined by the functions $z=Z(\zeta), w=W(\zeta)$. Then the following are equivalent:

(i) The embedding $\psi$ is totally real.

(ii) For each $\zeta \in U$ the equality $\mathbb{C} \cdot \psi_{*}\left(T_{\zeta} U\right)=T \mathbb{C}^{2}$ holds ${ }^{1}$.

(iii) The determinant $\left|\begin{array}{ll}\frac{\partial Z}{\partial \zeta} & \partial Z / \partial \bar{\zeta} \\ \frac{\partial W}{\partial \zeta} & \partial W / \partial \bar{\zeta}\end{array}\right|$ never vanishes.

(iv) The determinant $\left|\begin{array}{ll}\frac{\partial Z}{\partial u} & \frac{\partial Z}{\partial v} \\ \frac{\partial W}{\partial u} & \frac{\partial W}{\partial v}\end{array}\right|$ never vanishes.

Remark 1. Condition (ii) can be restated as follows. Let $\mathbf{X}=$ $\left(\mathrm{X}_{1}, \mathrm{X}_{2}\right)$ be a basis for the tangent space $T_{\zeta} U, \zeta \in U$. Then the vectors $\psi_{*}\left(\mathbf{X}_{1}\right)$ and $\psi_{*}\left(\mathbf{X}_{2}\right)$ form a complex basis for the complex vector space $T_{\psi(\zeta)} \mathbb{C}^{2}$

We introduce the notation $A_{a}^{-}=\{\zeta: 1-a<|\zeta| \leq 1\}$ and $A_{a}^{+}=$ $\{\zeta: 1 \leq|\zeta| \leq 1+a\}$ for $a>0$. The special case in which $\psi$ is defined on the annular region $A_{a}^{+}, a>0$ and $\psi$ is of the form

$$
\psi(\zeta)=(\zeta, f(\zeta)), \quad f(\zeta)=0 \text { for }|\zeta|=1
$$

is of particular interest to us. First observe that condition (iii) reduces to

$$
\frac{\partial f}{\partial \tilde{\zeta}} \neq 0
$$

It will prove useful to write equation (1) in polar coordinates, $z=r e^{i \theta}$,

$$
\frac{\partial f}{\partial \theta}-i r \frac{\partial f}{\partial r} \neq 0
$$

Because $f(z)$ vanishes for all $|z|=1$, it follows that $\frac{\partial f}{\partial \theta}=0$ and total reality implies that $\frac{\partial f}{\partial r} \neq 0$ for all $z$ with $|z|=1$. This implics the following lemma.

Lemma 2. Let $\psi$ be a totally real embedding of the annulus $A$ as given above. Then there is an annular region of the form $1 \leq|z|<1+a^{\prime}$, $0<a^{\prime}<a<1$, on which $f$ can we written in the form

$$
f(z)=R(z) e^{i \Theta(z)},
$$

IIf $V \subset W$ is a real subspace of the complex vector space $W$ then $\mathbb{C} \cdot V$ denotes the complex subspace spanned by $V$. 
where $R(z)$ is a non-negative, smooth real-valued function which vanishes for $|z|=1$ and where $\Theta(z)$ is smooth modulo $2 \pi$.

The inequality $\frac{\partial R(z)}{\partial r}>0$ is satisfied for all $z$ in a neighborhood of the unit circle $|z|=1$.

Now suppose that $f$ is any smooth function defined on $A$ of the form

$$
f(z)=R(z) e^{i \Theta(z)}
$$

Substitution of the formulas

$$
\frac{\partial f}{\partial r}=\left(\frac{\partial R(z)}{\partial r}+i R(z) \frac{\partial \Theta}{\partial r}\right) e^{i \Theta} \text { and } \frac{\partial f}{\partial \theta}=\left(\frac{\partial R(z)}{\partial \theta}+i R(z) \frac{\partial \Theta}{\partial \theta}\right) e^{i \Theta}
$$

into the total reality condition (2) and separating real and imaginary parts of the coefficient of $e^{i \Theta}$ yields the condition

$$
\left(\frac{\partial R(z)}{\partial \theta}+R(z) r \frac{\partial \Theta}{\partial r}\right)+i\left(R(z) \frac{\partial \Theta}{\partial \theta}-r \frac{\partial R(z)}{\partial r}\right) \neq 0 .
$$

In particular, if the imaginary part of the left hand side is negative, the embedding is necessarily totally real.

Lemma 3. Let $\psi: A_{a}^{+} \rightarrow \mathbb{C}^{2}, 0<a<1$, be an embedding of the form $\psi(z)=\left(z, R(z) e^{i \Theta(z)}\right)$, where $R$ and $\Theta$ are real-valued functions with $R$ smooth and $\Theta$ smooth modulo $2 \pi$. If the inequality

$$
r \frac{\partial R(z)}{\partial r}>R(z) \frac{\partial \Theta}{\partial \theta}
$$

is satisfied then the embedding is totally real.

2.2. An index for totally real embeddings of annuli. In this section we define an index for a totally real embedding of an annulus in $\mathbb{C}^{2}$. It is closely related to the Maslov index and is a special case of an index defined by Kamber and Tondeur [3]. A detailed presentation, within the context of totally real embeddings of surfaces in $\mathbb{C}^{2}$, is given in [2]. We give a self-contained exposition here.

Let $\phi_{0}: A \rightarrow \mathbb{C}^{2}$ be any totally real embedding of an annular region $A \subset \mathbb{C}$. To define the index of $\phi_{0}$ begin by choosing a complex framing ${ }^{2}$

${ }^{2}$ By a complex framing we mcan a pair of complex vector fields which are pointwise independent over $\mathbb{C}$. 
$\mathrm{f}=\left(\mathrm{f}_{1}, \mathrm{f}_{2}\right)$ for the holomorphic tangent bundle $T_{(1,0)} \mathcal{U}$, where $\mathcal{U}$ is any open contractible neighhborhood of $A$.

Next choose a real framing ${ }^{3} \mathbf{X}=\left(\mathbf{X}_{1}, \mathbf{X}_{2}\right)$ of the real tangent bundle $T A$ which is compatible with the orientation of $A$ as a subset of $\mathbb{C}$. By virtue of Remark 1, there is a smooth matrix-valued function

$$
M_{\phi_{0}}: A \rightarrow \mathrm{GL}(2, \mathbb{C})
$$

defined by the formula

$$
\left(\begin{array}{ll}
d \phi_{0}\left(\mathbf{X}_{1}\right) \quad d \phi_{0}\left(\mathbf{X}_{2}\right)
\end{array}\right)=\left(\begin{array}{ll}
\mathbf{f}_{1} & \mathbf{f}_{2}
\end{array}\right)\left(\begin{array}{ll}
m_{1}^{1} & m_{2}^{1} \\
m_{1}^{2} & m_{2}^{2}
\end{array}\right) .
$$

Definition 1 . The index of the embedding $\phi_{0}: A \rightarrow \mathbb{C}^{2}$, is the degree of the map

$$
A \rightarrow \partial \Delta: \zeta \mapsto \frac{\operatorname{det}(M)}{|\operatorname{det}(M)|}
$$

and is denoted by Ind $\left(\phi_{0}\right) \in \mathbb{Z}$.

Remark 2. (i) It is easily verified that the integer $\operatorname{Ind}\left(\phi_{0}\right)$ is independent of the framings $X$ and $f$. For suppose that $X^{\prime}$ and $f^{\prime}$ is another pair of framings, with $\mathrm{f}^{\prime}$ defined on $\mathcal{U}^{\prime}$, a contractible neighborhood of $\phi_{0}(A)$. Then there are smooth maps $B: \mathcal{U} \cap \mathcal{U}^{\prime} \rightarrow \mathrm{GL}(2, \mathbb{C})$ and $C: A_{\epsilon}^{-} \rightarrow \mathrm{GL}^{+}(2, \mathbb{R})$ such that

$$
\mathbf{f}^{\prime}=\mathbf{f} \cdot B \quad \text { and } \quad \mathbf{X}^{\prime}=\mathbf{X} \cdot C .
$$

If $M^{\prime}: A \rightarrow \mathrm{GL}(2, \mathbb{C})$ is the map defined by the formula $d \phi_{0}(\mathbf{X})=\mathrm{f} \cdot M^{\prime}$, a straightforward calculation with matrices yields the identity

$$
M^{\prime}=B^{-1} \cdot M \cdot C .
$$

Since $C$ is real, so is $\operatorname{det}(C)$, hence,

$$
\frac{\operatorname{det}\left(M^{\prime}\right)}{\left|\operatorname{det}\left(M^{\prime}\right)\right|}=\frac{\operatorname{det}\left(B^{-1}\right) \operatorname{det}(M) \operatorname{det}(C)}{\left|\operatorname{det}\left(B^{-1}\right)\right||\operatorname{det}(M)||\operatorname{det}(C)|}=\frac{|\operatorname{det}(B)|}{\operatorname{det}(B)} \frac{\operatorname{det}(M)}{|\operatorname{det}(M)|} .
$$

Since $\mathcal{U}$ and $\mathcal{U}^{\prime}$ are contractible, each of the framings $\mathbf{f}$ and $\mathrm{f}^{\prime}$ are is homotopic to the framing $\left(\frac{\partial}{\partial z^{1}}, \frac{\partial}{\partial z^{2}}\right)$. The map $B^{-1}$ is, therefore, homotopic to the identity. This fact, together with the observation that the

\footnotetext{
${ }^{3}$ By a real framing we mean a pair of real vector fields which are pointwise independent over $\mathbb{R}$.
} 
degree depends only on the homotopy class of the map, completes the argument.

(ii) Note also that the above argument shows that if $\Phi: \mathcal{U} \rightarrow \mathbb{C}^{2}$ is a biholomorphism onto an open set in $\mathbb{C}^{2}$ then

$$
\operatorname{Ind}\left(\Phi \circ \phi_{0}\right)=\operatorname{Ind}\left(\phi_{0}\right)
$$

(iii) Finally, because the index depends only on the homotopy class of the map $\phi_{0}$, it is determined by the image $\phi_{0}(A)$ together with an orientation. Thus, if $A \subset \mathbb{C}^{2}$ is a totally real, oriented, embedded annulus, the integer $\operatorname{Ind}(A)$ is well-dcfined.

Lemma 4. If $A \subset \mathbb{C}^{2}$ is a totally real embedded onnulus which is contained in a totally real embedded disk $D \subset \mathbb{C}^{2}$ then $\operatorname{Ind}(A)=0$.

Proof: Let $\phi: \Delta \rightarrow \mathbb{C}^{2}$ a smooth map such that $\phi(\Delta)=D$ and choose framings $\mathrm{X}$ of $T \Delta$ and $\mathrm{f}$ of $\mathbb{C}^{2}$. Then let $M(\zeta), \zeta \in \Delta$, be the $\mathrm{GL}(2, \mathbb{C})$-valued matrix as defined above. The degree of the map $\zeta \rightarrow \operatorname{det}(M(\zeta)) /|\operatorname{det}(M(\zeta))| \zeta \in \phi^{-1}(A)$ is zero because it is homotopic to a constant.

\section{Reduction to the case of real analytic boundary}

Begin by assuming that there are smooth embeddings $\psi$ and $\phi$ of $\bar{\Delta}$ with $\psi$ holomorphic on $\Delta$ and $\phi$ totally real and such that the conditions $\psi(\Delta) \cap \phi(\Delta)=\emptyset$ and $\psi(\partial \Delta)=\phi(\partial \Delta)$ are both satisfied.

Without loss of generality we may assume that $\psi$ extends holomorphically to a neighborhood of $\bar{\Delta}$. To see this we observe that, because the condition of total reality is an open condition, any $C^{1}$-small deformation of the map $\phi$ is also a totally real embedding. In particular, let

$$
\partial \Delta_{\delta}=\{\zeta:|\zeta|=1-\delta\}
$$

where $\delta>0$ is a small constant to be chosen later. Then $\phi$ can be deformed to a map $\phi^{\prime}$ so that

$$
\phi^{\prime}(\partial \Delta)=\psi\left(\partial \Delta_{\delta}\right)
$$

and in such a manner that

$$
\phi(\Delta) \cap \psi\left(\Delta_{\delta}\right)=\emptyset
$$


One way to accomplish such a deformation is to let $\phi^{\prime}$ be the composition $\phi \circ \nu_{\delta}$ of $\phi$ with the flow, $\nu_{t}$, of a vector ficld which is tangent to the image of $\psi$ and constructed so that $\nu_{\delta}\left(\psi(\partial \Delta)=\psi\left(\partial \Delta_{\delta}\right), \delta>0\right.$.

The map $\psi^{\prime}: \Delta \rightarrow \mathbb{C}^{2}$ defined by the equation

$$
\psi^{\prime}(\zeta)=\psi((1-\delta) \zeta)
$$

extends holomorphically to a ncighborhood of $\dot{\Delta} \subset \mathbb{C}$. Now replace the pair $\psi, \phi$ by the pair $\psi^{\prime}, \phi^{\prime}$. By construction, $\psi(\partial \Delta)$ is real analytic.

\section{Holomorphic disks are relatively isotopic to totally real disks}

Return now to the problem of replacing $\psi$ by a totally real embedding. Because $\psi$ extends to a holomorphic embedding of a neighborhood of $\bar{\Delta}$, there is a biholomorphism $\Phi: \mathcal{U} \rightarrow \mathbb{C}^{2}$, defined on a neighborhood $\mathcal{U}$ of $\psi(\bar{\Delta})$ such that the composition $\Phi \circ \psi$ is the map

$$
\zeta \mapsto(\zeta, 0) .
$$

Consider the family of maps

$$
\psi_{c}^{\prime}: \Delta \rightarrow \mathbb{C}^{2}, \quad \zeta \mapsto(z, w)=\left(\zeta, f_{\epsilon}(\zeta)\right)
$$

where

$$
f_{\epsilon}(\zeta)=\epsilon\left(1-|\zeta|^{2}\right) e^{i\left(1-|\zeta|^{2}\right)} \bar{\zeta}
$$

Note that $f_{\epsilon}$ satisfies the conditions: $f_{0}=0, f_{\epsilon}(\zeta)=0$ for $|\zeta|=1$. By virtue of equation (1) and the computation

$$
\frac{\partial f_{\epsilon}(\zeta)}{\partial \vec{\zeta}}=\epsilon\left\{\left(1-2|\zeta|^{2}\right)-\left(1-|\zeta|^{2}\right)|\zeta|^{2} i\right\} e^{i\left(1-|\zeta|^{2}\right)} \neq 0
$$

the embedding defined by $\psi_{\epsilon}^{\prime}$ is totally real for all $\epsilon>0$.

Because for $\epsilon$ sufficiently small the image of $\psi_{\epsilon}^{\prime}$ lies in the set $\Phi(\mathcal{U})$, the map $\dot{\psi}_{\epsilon}=\Phi^{-1} \circ \psi_{\epsilon}^{\prime}$ is well-defined.

Lemma 5. For 6 sufficiently small, the family $\psi_{\epsilon}$ has the following properties:

(i) $\psi_{\epsilon \mid \partial \Delta}=\psi_{\mid \partial \Delta}$ for all $\epsilon$.

(ii) $\psi_{\epsilon}$ is a totally real embedding of $\bar{\Delta}$ for $\epsilon>0$.

(iii) $\psi_{\epsilon}(\bar{\Delta}) \subset \mathcal{U}$.

(iv) $\psi_{\epsilon}(\Delta) \cap \phi(\Delta)=\emptyset$. 
Proof: Properties (i) through (iii) are immediate from the definition of $f_{\epsilon}$.

The verification of (iv) is based on the implicit function theorem. First note that since $\phi(\bar{\Delta})$ is totally real and $\psi_{0}(\bar{\Delta})$ is holomorphic, $T_{p}\left(\psi_{0}(\bar{\Delta})\right) \cap T_{p}(\phi(\bar{\Delta}))=T_{p}(\psi(\partial \Delta))$ for all $p \in \psi(\partial \Delta)$. Because $\psi_{\varepsilon}$ depends smoothly on $\epsilon$, the condition $T_{p}\left(\psi_{\epsilon}(\bar{\Delta})\right) \cap T_{p}(\phi(\bar{\Delta}))=T_{p}(\psi(\partial \Delta))$ holds for all $\epsilon$ sufficiently small. By the implicit function theorem and compactness of $\partial \Delta$, it follows that there is a number $\delta>0$ such that $\psi_{\epsilon}(\Delta) \cup \phi\left(A_{\delta}^{-}\right)=\emptyset$ for all sufficiently small $\epsilon$. Moroeover, since $\psi_{0}(\Delta) \cap \phi(\Delta)=\emptyset$ and $\psi_{\epsilon}$ depends smoothly on $\epsilon$, it follows from the compactness of $\phi\left(\Delta \backslash A_{\delta}^{-}\right)$that $\psi_{\varepsilon}(\Delta) \cap \phi\left(\Delta \backslash A_{\delta}^{-}\right)=\emptyset$ for all sufficiently small $\epsilon$. Hence, for $\epsilon$ sufficiently small, $\psi_{\epsilon}(\Delta) \cap \phi(\Delta)=\emptyset$.

\section{Modifying two totally real disks to abut smoothly}

Consider the small annular neighborhood $A_{\delta}^{-} \subset \bar{\Delta}, 0<\delta<1$, of the boundary $\partial \Delta$. We will modify $\phi(\bar{\Delta})$ on $\phi\left(A_{\delta}^{-}\right)$so that $\phi(\bar{\Delta})$ and $\psi_{\epsilon}(\bar{\Delta})$ abut in a $C^{1}$ manner along $\phi(\partial \Delta)$ and thus define a totally real embedding of the 2-sphere into $\mathbb{C}^{2}$.

By virtue of the equality $\psi(\partial \Delta)=\phi(\partial \Delta)$, for $\delta$ sufficiently small the irnage $\phi\left(A_{\delta}^{-}\right)$is contained in the neighborhood $\mathcal{U}$ of the previous section. For this reason the map

$$
\phi^{\prime}=\Phi \circ \phi: A_{\hat{\delta}}^{-} \rightarrow \mathbb{C}^{2}
$$

is well-defined and, since we will modify $\phi$ only along $A_{\delta}^{-}$, the deformation of $\phi$ can be reinterpreted as a deformation of $\phi^{\prime}$. The modification will be done in two stages: (i) we first deform $\phi^{\prime}$ so that $\phi^{\prime}\left(A_{\delta}^{-}\right)$is the graph of a function $g$; (ii) then we deform $g$ so that $\psi(\Delta)$ and $\phi(\Delta)$ abut smoothly.

5.1. Replacing $\phi^{\prime}\left(A_{\delta}^{-}\right)$by the graph of a function. Consider the neighborhoods of $\psi^{\prime}(\bar{\Delta})$ of the form

$$
\mathcal{N}_{\epsilon, \sigma}=\{(z, w):|z|<1+\epsilon,|w|<\sigma\}
$$

with $\epsilon>0$ and $\sigma>0$ chosen so small that there is an inclusion $\mathcal{N}_{3 \epsilon, \sigma} \subset$ $\Phi(\mathcal{U})$ and such that the condition

$$
\Phi^{-1}\left(\mathcal{N}_{3 \epsilon, \sigma}\right) \cap \phi\left(\Delta \backslash A_{\delta}^{-}\right)=\emptyset
$$

is satisfied. By choosing $\delta^{\prime}<\delta$ sufficiently small we can insure that inclusion $\phi^{\prime}\left(A_{\delta^{\prime}}^{-}\right) \subset \mathcal{N}_{\epsilon, \sigma}$ is satisfied. 
We are going to replace $\phi^{\prime}$ by another totally real embedding $\phi^{\prime \prime}$ : $A_{\delta}^{-} \rightarrow \mathcal{N}_{2 \varepsilon, \sigma}$ which satisfies the two conditions:

$$
\phi^{\prime \prime}=\phi^{\prime} \text { on the annular region } A_{\delta}^{-} \backslash A_{\delta^{\prime}}^{-}
$$

and

$$
\phi^{\prime \prime}\left(A_{\delta}^{-}\right) \cap \mathcal{N}_{\epsilon, \sigma^{\prime}}=\left\{(z, g(z)): z \in A_{\sigma^{\prime}}^{+}\right\} \text {for } \sigma^{\prime}<\sigma \text { sufficiently small, }
$$

where $g$ is a complex-valued function defined on $A_{\sigma^{t}}^{+}$. By construction, the map $\Phi^{-1} \circ \phi^{\prime \prime}: A_{\delta}^{-} \rightarrow \mathbb{C}^{2}$ agrees with $\phi$ on the interior boundary component of the annulus and so defines another totally real embedding, $\phi_{1}: \Delta \rightarrow \mathbb{C}^{2}$ which intersects $\psi(\Delta)$ along the circle $\psi(\partial \Delta)$.

To begin the construction of $\phi^{\prime \prime}$ observe that the image $\phi^{\prime}\left(A_{\delta}^{-}\right)$is of the form

$$
z=Z(\zeta), \quad w=W(\zeta), \quad 1-\delta \leq|\zeta|<1,
$$

where the functions $Z$ and $W$ satisfy the conditions

$$
Z(\zeta)=\zeta \text { and } W(\zeta)=0 \text { for }|\zeta|=1
$$

Hence, in polar coordinates $\zeta=\rho e^{i \alpha}, z=r e^{i \theta}$ we can write

$$
Z=R(\rho, \alpha) e^{i \Theta(\rho, \alpha)}
$$

with $R(1, \alpha)=1$ and $\Theta(1, \alpha)=\alpha$. Applying condition (iv) of Lemma 1 yields the inequality

$$
0 \neq\left|\begin{array}{ll}
\frac{\partial Z}{\partial \rho} & \frac{\partial Z}{\partial \alpha} \\
\frac{\partial W}{\partial \rho} & \frac{\partial W}{\partial \alpha}
\end{array}\right|=\left|\begin{array}{cc}
\frac{\partial Z}{\partial \rho} & \frac{\partial R e^{i \Theta}}{\partial \alpha} \\
\frac{\partial W}{\partial \rho} & 0
\end{array}\right|=-i e^{i \alpha} \frac{\partial W}{\partial \rho} \quad \text { when } \rho=1
$$

Thus $\frac{\partial W}{\partial \rho}$ is non-zero for $(\rho, \alpha)=(1, \alpha)$. Continuity implies that for $\delta^{\prime}>0$ sufficiently small $\frac{\partial W}{\partial \rho}$ does not vanish anywhere on the annulus $A_{\delta}^{-}$. This and equation (4) show that, after possibly decreasing $\delta^{\prime}$ still further, the map $\zeta \mapsto\left(e^{i \Theta}, W\right)$ is an embedding of $A_{\delta^{\prime}}^{-}$; and, therefore, that for any smooth function $\tilde{R}(\rho, \alpha)>0$ the map

$$
\phi^{\prime \prime}: \zeta \mapsto(\tilde{Z}(\zeta), W(\zeta))=\left(\tilde{R} e^{i \Theta}, W\right)
$$

defines an embedding of $A_{\delta^{\prime}}^{-}$. Of course, we wish to choose $\vec{R}$ so that the map $\phi^{\prime \prime}$ satisfies the conditions stated above. That we can do so is implied by the following lemma. 
Lemma 6 . There exists a function $\tilde{R}$ such that $\phi^{\prime \prime}$ satisfies the conditions:

(6) $\quad \tilde{R}(\rho, \alpha)=R(\rho, \alpha)$ for $\rho<1-\delta^{\prime}$.

(7) $\quad \tilde{R}(1, \alpha)=1$.

$$
\frac{\partial \tilde{Z}}{\partial \rho} \frac{\partial W}{\partial \alpha}-\frac{\partial W}{\partial \rho} \frac{\partial \bar{Z}}{\partial \alpha} \neq 0 \text { when } \rho \leq 1 \quad \text { (total reality). }
$$$$
\vec{R}(1, \alpha)=1 \text {. }
$$$$
|\tilde{R}(\rho, \alpha)-1|<2 \epsilon \text { for } 1-\delta^{\prime}<\rho \leq 1 \text {. }
$$$$
\frac{\partial \hat{R}(\rho, \alpha)}{\partial \rho}<0 \text { for } 1-\rho \text { sufficiently small. }
$$

\section{Remark 3.}

Condition (5) is the total reality condition.

Conditions (6-8) imply that the formula

$$
\phi_{1}(\zeta)= \begin{cases}\phi(\zeta) & \text { for }|\zeta|<1-\delta^{\prime} \\ \Phi^{-1} \circ \phi^{\prime \prime}(\zeta) & \text { otherwise }\end{cases}
$$

defines an embedding of $\Delta$ such that

$$
\begin{aligned}
& \phi_{1}(\Delta) \cap \psi(\Delta)=\emptyset \\
& \phi_{1}(\partial \Delta)=\psi(\partial \Delta) .
\end{aligned}
$$

Condition (8) also insures that $\phi_{1}\left(A_{\delta^{\prime}}^{-}\right)$is contained within the open set $\Phi^{-1}\left(\mathcal{N}_{2 \epsilon, \sigma}\right)$, and it implies that the intersection $\phi_{1}(\Delta) \cap \psi\left(A_{\delta}^{-}\right)$is empty.

Condition (9) insures that the intersection $\phi^{\prime}\left(A_{\delta}^{-}\right) \cap \mathcal{N}_{\epsilon, \sigma^{\prime}}$ coincides with the graph of a function $g$ over the annulus $A_{\sigma^{\prime}}^{+}$, for sufficiently small $\sigma^{\prime}>0$

Proof of Lemma 6: Let $b=2 \max _{\zeta \in A_{\dot{b}}^{\prime}}\left|\frac{\partial R(\zeta)}{\partial \rho}\right|+1$ and $\tilde{R}(\zeta)=R(\zeta)+$ $b h(\rho)(1-\rho)$, where $h$ is a real valued, non-decreasing function such that

$$
\begin{aligned}
h(\rho) & = \begin{cases}1 & \text { for } 1-\delta^{\prime \prime} / 8<\rho \leq 1 \\
0 & \text { for } \rho<I-7 \delta^{\prime \prime} / 8\end{cases} \\
0 & \leq h^{\prime}(\rho) \leq 2 / \delta^{\prime \prime}
\end{aligned}
$$

and $\delta^{\prime \prime}<\delta^{\prime} / 2<\epsilon$ is to be determined. (We Icave it to the reader to show that such a function exists.) Conditions (6) and (7) are easily verified.

To check that property (8) is satisfied, note that choosing $\delta^{\prime \prime}<\epsilon / b$ gives the estimate

$$
|b h(\rho)(1-\rho)| \leq b 7 \delta^{\prime \prime} / 8<\epsilon,
$$


from which the inequality,

$$
|\tilde{R}(\zeta)|<|R(\zeta)|+b 7 \delta^{\prime \prime} / 8<|R(\zeta)|+\epsilon
$$

easily follows. Next observe that by virtue of equation (4) and the continuity of $R$, by choosing $\delta^{\prime}$ sufficiently small we can insure that the inequality $|R(\zeta)-1| \leq \epsilon$ is satisfied for $\zeta \in A_{\delta^{\prime}}^{-}$. This yields the desired inequality $(8)$.

To prove that the inequality (9) is satisfied, first note that for $1-\rho<$ $\delta^{\prime \prime} / 8, h(\rho)=1$ and $h^{\prime}(\rho)=0$. Thus,

$$
\frac{\partial \bar{R}}{\partial \rho}=\frac{\partial R}{\partial \rho}-b<0
$$

It remains to show that for $\delta^{\prime \prime}$ sufficiently small the condition of total reality is satisfied for $\rho>1-\delta^{\prime \prime}$. It suffices to show that the expression $\frac{\partial \bar{Z}}{\partial \alpha} \frac{\partial W}{\partial \rho}-\frac{\partial \bar{Z}}{\partial \rho} \frac{\partial W}{\partial \alpha}$ is positive. Compute as follows:

$$
\begin{aligned}
& \frac{\partial \tilde{Z}}{\partial \alpha} \frac{\partial W}{\partial \rho}-\frac{\partial \tilde{Z}}{\partial \rho} \frac{\partial W}{\partial \alpha}= \frac{\partial\left(Z+b h(\rho)(1-\rho) e^{i \Theta}\right)}{\partial \alpha} \frac{\partial W}{\partial \rho} \\
&-\frac{\partial\left(Z+b h(\rho)(1-\rho) e^{i \Theta}\right)}{\partial \rho} \frac{\partial W}{\partial \alpha} \\
&=\frac{\partial Z}{\partial \alpha} \frac{\partial W}{\partial \rho}-\frac{\partial Z}{\partial \rho} \frac{\partial W}{\partial \alpha} \\
&+i\left(b h(\rho)(1-\rho) e^{i \Theta}\right) \frac{\partial \Theta}{\partial \alpha} \frac{\partial W}{\partial \rho} \\
&-\frac{\partial\left(b h(\rho)(1-\rho) e^{i \Theta}\right)}{\partial \rho} \frac{\partial W}{\partial \alpha} .
\end{aligned}
$$

It suffices to show that the expression $\frac{\partial \bar{Z}}{\partial \alpha} \frac{\partial W}{\partial \rho}-\frac{\partial \dot{Z}}{\partial \rho} \frac{\partial W}{\partial \alpha}$ is positive. Consider the inequality,

$$
\begin{gathered}
\left|\frac{\partial \dot{Z}}{\partial \alpha} \frac{\partial W}{\partial \rho}-\frac{\partial \dot{Z}}{\partial \rho} \frac{\partial W}{\partial \alpha}\right|>\left|\frac{\partial Z}{\partial \alpha} \frac{\partial W}{\partial \rho}-\frac{\partial Z}{\partial \rho} \frac{\partial W}{\partial \alpha}\right|-\left|b h(\rho) \frac{\partial \Theta}{\partial \alpha} \frac{\partial W}{\partial \rho}\right||1-\rho| \\
-\left|\frac{\partial\left(b h(\rho)(1-\rho) e^{i \Theta(\rho, \alpha)}\right)}{\partial \rho}\right|\left|\frac{\partial W}{\partial \alpha}\right| .
\end{gathered}
$$

The total reality of the original embedding $(\rho, \alpha) \mapsto(Z(\rho, \alpha), \Theta(\rho, \alpha))$ guarantees that the first term is bounded below by a positive constant for $1-\delta^{\prime \prime} \leq \rho \leq 1$. 
By choosing $\delta^{\prime \prime}$ small the second term can be made arbitrarily small.

Finally, consider the third term. Recall that the equality $W=0$ holds along the circle $\rho=1$ and that $W$ is a smooth function of $\rho$ and $\alpha$. Also recall the $h^{\prime}(\rho)$ is bounded above by $2 / \delta^{\prime \prime}$, so that $\left|h^{\prime}(\rho)(1-\rho)\right|<2$; this, in turn, implies that the coefficient of $\left|\frac{\partial W}{\partial \alpha}\right|$ is bounded by a constant which is independent of $\delta^{\prime \prime}$. But for $\rho$ sufficiently near 1 , the partial derivative $\partial W / \partial \alpha$ can be made arbitrarily small. Thus, for $\delta^{\prime \prime}$ sufficiently small, the third term can be made arbitrarily small and the right hand side positive.

We have verified the total reality condition for $\rho<1-\delta^{\prime \prime}$. To verify it for $\rho \geq 1-\delta^{\prime \prime}$, notice that the equality $\phi^{\prime \prime}=\phi^{\prime}$ holds for $\rho \geq 1-\delta^{\prime \prime}$ and that $\phi^{\prime}$ is totally real.

5.2. Smoothly joining $\psi(\Delta)$ and $\phi(\Delta)$. By the results above, we may assume that $\phi$ has been replaced by $\phi_{1}$ and $\delta$ by $\delta^{\prime}$ and that $\epsilon$ and $\sigma$ have been shrunk so that the following conditions are satisfied:

$$
\begin{gathered}
\Phi \circ \phi\left(A_{\delta}^{-}\right) \cap \mathcal{N}_{\epsilon, \sigma}=\left\{(z, g(z)): z \in A_{\sigma}^{+}\right\} \\
\psi\left(\Delta \backslash A_{\delta}^{-}\right) \cap \Phi^{-1}\left(\mathcal{N}_{\epsilon, \sigma}\right)=\emptyset .
\end{gathered}
$$

The next step is to modify the function $g$ on a neighborhood of the unit circle so that it agrees with $f_{\varepsilon}$.

Begin by extending $f_{\epsilon}$ to the disk of radius $1+\sigma$ using formula (3); and observe it has the polar form

$$
f_{\epsilon}(z)=\epsilon\left(r^{2}-1\right) r e^{i\left(\pi+1-r^{2}-\theta\right)}
$$

on the annulus $A_{\sigma}^{+}$. Notice the addition of the term $i \pi$ in the exponent, and the sign change in the modulus (c.f. equation (3)).

Lemma 7. There exist real numbers $\sigma^{\prime}$ and $\sigma^{\prime \prime \prime}$ with $0<\sigma^{\prime \prime \prime}<\sigma^{\prime}<\sigma$ and a complex valued function $\bar{g}$, defined on the annulus $A_{\delta}^{+}$, which has the following properties

(i) $|\tilde{g}(z)|<\epsilon$ for all $z \in A_{\sigma}^{+}$.

(ii) $\frac{\partial \bar{g}(x)}{\partial \bar{z}} \neq 0$ for all $z \in A_{\sigma}^{+}$.

(iii) $\tilde{g}(z)=g(z)$ for $|z|>1+\sigma^{\prime}$.

(iv) $\tilde{g}(z)=f_{\epsilon}(z)$ for $1 \leq|z|<1+\sigma^{\prime \prime \prime}$.

Remark 4. Assume for the moment that Lemma 7 has been verified. We may then define a totally real embedding $\phi_{2}$ of the disk $\Delta_{1+\sigma}$ of radius $1+\sigma$ into $\mathbb{C}^{2}$ in the following way. Define $f: \Delta_{1+\sigma} \rightarrow \mathcal{N}_{\epsilon, \sigma}$ by

$$
f(z)= \begin{cases}f_{\epsilon}(z) & \text { for }|\zeta| \leq 1, \\ \tilde{g}(z) & \text { for } 1<|z|<1+\sigma .\end{cases}
$$


Then set $\phi_{2}(z)=\Phi^{-1}(z, f(z))$. By construction, the totally real disks $\psi(\Delta)$ and $\phi_{2}\left(\Delta_{1+\sigma}\right)$ intersect smoothly to define a totally real embedding of the two-sphere into $\mathbb{C}^{2}$. As already remarked, such embeddings do not exist. We have, arrived at a contradiction, and thus proved the Intersection Theorem.

Remark 5. The proposition also follows from Gromov's theory of differential inequalities [6]. The proof we give here does not rely on Gromov's theory.

The proof of the proposition proceeds in two steps:

Step 1. Construct $\tilde{g}$ so that it satisfies the conditions,

$$
\left\{\begin{array}{ll}
\arg (\tilde{g}(z))=\arg \left(f_{\epsilon}(z)\right) & \text { for } 1 \leq|z|<1+\sigma^{\prime \prime} \\
\arg (\tilde{g}(z)=\arg (g(z)) & \text { for }|z|>1+\sigma^{\prime} \\
|\bar{g}(z)|=|g(z)| & \text { for all } z,
\end{array},\right.
$$

where $\sigma^{\prime}$ and $\sigma^{\prime \prime}$ satisfy the inequalities $0<\sigma^{\prime \prime}<\sigma^{\prime}<\sigma$.

Step 2. Modify $\tilde{g}(z)$ on the set $1 \leq|z|<\sigma^{\prime \prime}$ so that, in addition to the conditions specified in Step 1, it satisfies the equality

$$
|\tilde{g}(z)|=\left|f_{\epsilon}(z)\right| \quad \text { for } 1 \leq|z|<\sigma^{\prime \prime \prime}<\sigma^{\prime \prime} .
$$

Step 1. Recall that by Lemma 2 we can write $g$ in the form $g(z)=$ $R(z) e^{i \Theta(z)}$, where $R(z)$ and $\Theta(z)$ are as in the lemma. We will write $\tilde{g}$ in the form

$$
\tilde{g}(z)=R(z) e^{i \check{\Theta}(z)},
$$

where $\tilde{\Theta}$ is to be determined.

Lemma 8. There is a smooth homotopy $H: \begin{cases}{[0,1] \times A_{\sigma}^{+}} & \rightarrow \partial \Delta \\ (t, z) & \mapsto H_{t}(z)\end{cases}$ such that $H_{0}=e^{i a r g\left(f_{e}\right)}$ and $H_{1}=e^{i \Theta}$.

Proof: By Remark 1 and Lemma 4 the index of the totally real embedding of $A_{\sigma}^{+}$, defined by $\zeta \mapsto(\zeta, g(\zeta))$, is zero.

On the other hand, the index equals the degree of the map

$$
\gamma: r e^{i \theta} \mapsto \operatorname{det}(M) /|\operatorname{det}(M)|
$$

where

$$
\begin{aligned}
M(r, \theta) & =\operatorname{det}\left(\begin{array}{ll}
\frac{\partial z}{\partial r} & \frac{\partial g(z)}{\partial r} \\
\frac{\partial z}{\partial \theta} & \frac{\partial g(z)}{\partial \theta}
\end{array}\right)=\operatorname{det}\left(\begin{array}{ll}
\frac{\partial\left(r e^{i \theta}\right)}{\partial r} & \frac{\partial g(z)}{\partial r} \\
\frac{\partial\left(r e^{i \theta}\right)}{\partial \theta} & \frac{\partial g(z)}{\partial \theta}
\end{array}\right) \\
& =e^{i \theta} \frac{\partial g(z)}{\partial \theta}-i r e^{i \theta} \frac{\partial g(z)}{\partial r} .
\end{aligned}
$$


Observe that the degree of $\gamma$ is the same as the degree of its restriction to the circle $\partial \Delta$. Since $\partial g / \partial \theta=R(r, \theta)=0$ and $\frac{\partial R(1, \theta)}{\partial r}>0$ on $\partial \Delta$, the degree of $y$ is the degree of the map

$$
e^{i \theta} \mapsto-i e^{i \theta} e^{i \Theta(1, \theta)} .
$$

But the degree of $\gamma$ is zero, hence the degree of the map $z \mapsto e^{i \Theta(1, \theta)}$ equals -1 .

Since the degree of the map $z \mapsto e^{i a r g\left(f_{\epsilon}(z)\right.}=e^{i\left(\pi+1-r^{2}-\theta\right)}$ is also equal to -1 , the two maps are homotopic.

Next let $h_{s}(r)$ be a smooth family of monotone, non-decreasing functions such that (i) $h_{s}(r)=0$ for $r \leq s / 3$, and (ii) $h_{s}(r)=1$ for $r \geq 2 s / 3$. The map $g_{s}$ is then defined by the equation

$$
g_{s}(z)=R(z) H_{h_{9}(|z|)}(z) .
$$

That the modulus of $g_{s}$ is bounded by $\epsilon$ is clear from the fact that $g_{s}$ was obtained from $g$ by only changing its argument; thus, $\left|g_{s}(z)\right|=$ $|g(z)| \leq \epsilon$.

We need only show that for $s$ sufficiently small, say $s=\sigma^{\prime \prime}$, the map $g_{s}$ defines a totally real embedding for $1 \leq r<1+\sigma$-all other properties that $\tilde{g}$ must satisfy are clear from its definition. Also, since $g_{s}(z)=g(z)$ for $|z| \geq 1+s$, we need only verify total reality for $1 \leq|z|<1+s$.

Write $H_{t}(z)$ in the form

$$
H_{t}(z)=e^{\Theta_{t}(z)}
$$

where $\Theta_{t}(z)$ is a smooth function modulo $2 \pi$ of $t$ and $z$. By virtue of Lemma 3 it suffices to prove that for $s$ sufficiently small the identity

$$
r \frac{\partial R(r, \theta)}{\partial r}>R(z) \frac{\partial \Theta_{h_{s}(r)}(r, \theta)}{\partial \theta}
$$

is satisfied for all $1 \leq r<1+s$.

Let $C>0$ and $c>0$ be constants such that the inequalities

$$
\begin{gathered}
\left|\frac{\partial \Theta_{t}(r, \theta)}{\partial \theta}\right|<C, \quad(t, r, \theta) \in[0,1] \times A_{\sigma}^{+} \\
\frac{\partial R(r, \theta)}{\partial r}>c, \quad 1 \leq r<1+s
\end{gathered}
$$

are satisfied (the existence of $c$ is implied by total reality). Because $R(r, \theta)$ is zero for $r=1$, for $s=\sigma^{\prime}$ sufficiently small the inequality $R(z)<\frac{c}{C}$ is satisfied, and a fortiori so is the inequality (10). 
Step 2. Assume now that $g$ has been replaced by $g_{s}$ and write

$$
g(z)=R(z) e^{i \Theta(z)}
$$

so that $\Theta(z)=\arg \left(f_{\epsilon}(z)\right)$ for $1 \leq|z|<1+\sigma^{\prime \prime}$.

Define a new function

$$
\tilde{g}(z)=\tilde{R}(z) e^{i \Theta(z)}
$$

where $\tilde{R}$ is of the form

$$
\tilde{R}(z)=(1-h(r))\left|f_{\epsilon}(z)\right|+h(r) R(z)
$$

and $h$ is a non-decreasing function with $h(r)=0$ for $r$ near $1, h(r)=1$ for $r>1+\sigma^{\prime \prime}$, and where $\sigma^{\prime \prime}: 0<\sigma^{\prime \prime}<\sigma^{\prime}$ will be selected shortly. The modulus of $\tilde{g}$ is then a convex combination of the moduli of $f_{\varepsilon}$ and $g$; thus $|\tilde{g}|$ is bounded by $\epsilon$.

It remains only to verify that $h$ can be chosen in such a way that the total reality condition is satisfied by $\tilde{g}$. By Lemma 3 the following lemma suffices.

Lemma 9. The function $h$ can be chosen is such a way that the inequality

$$
r \frac{\partial \tilde{R}(z)}{\partial r}>\tilde{R}(z) \frac{\partial \Theta}{\partial \theta}
$$

is satisfied for all $z \in A_{\sigma^{\prime \prime}}^{+}$.

Proof: By virtue of Lemma 2, and after a possible necessary reduction in the value of $\sigma^{\prime}$, there exist positive constants $m, M$ and $C$ such that the inequalities,

$$
\begin{gathered}
\left|\frac{\partial \Theta}{\partial \theta}\right|<C \quad m<\frac{\partial\left|f_{\epsilon}\right|}{\partial r}<M \quad m<\frac{\partial R}{\partial r}<M \\
m(r-1) \leq\left|f_{\epsilon}(z)\right| \leq M(r-1) \quad m(r-1) \leq|R(z)| \leq M(r-1)
\end{gathered}
$$

are satisfied for all $1 \leq|z| \leq 1+\sigma^{\prime}$.

The function $h$ will be selected so that the inequality $\frac{1}{\tilde{R}(z)} \frac{\partial \dot{R}(z)}{\partial r}>C$ is satisficd. Estimate as follows:

$$
\begin{aligned}
\frac{1}{\tilde{R}(z)} \frac{\partial \tilde{R} z}{\partial r} & \geq \frac{m-h^{\prime}(r)\left(\left|f_{\epsilon}(z)\right|-R(z)\right)}{M(r-1)} \\
& \geq \frac{m-(M-m)(r-1) h^{\prime}(r)}{M(r-1)}=\frac{m / M}{(r-1)}-\left(\frac{M-m}{M}\right) h^{\prime}(r) .
\end{aligned}
$$


Choose $\sigma^{\prime \prime}>0$ so that $\frac{m / M}{\sigma^{\prime \prime}}>C$. We claim that there is a constant $\sigma^{\prime \prime \prime}<$ $\sigma^{\prime \prime}$ and a function $h$ such that $h(r)=0$ for $1 \leq r<1+\sigma^{\prime \prime \prime}, h(r)=1$ for $r>1+\sigma^{\prime \prime} / 2$, and $h^{\prime}(r) \leq H(r)$ where $H(r)=\left(\frac{M-m}{M}\right)^{-1}\left(\frac{m / M}{(r-\overline{1})}-C\right)$. That such a function exists is clear because the right hand side of the last inequality is positive for $1<r<1+\sigma^{\prime \prime}$ and because the integral $\int_{1}^{1+\sigma^{\prime \prime}} H(r) d r$ diverges to $+\infty$. Hence we may set $h(r)=\int_{1}^{r} k(t) d t$ where $k(r)$ is any function satisfying the conditions, (i) $0 \leq k(r) \leq H(r)$, (ii) $\int_{1}^{\mathrm{I}+\sigma^{\prime \prime}} k(r) d r=1$, and (iii) $k(r)=0$ for $1 \leq r<\sigma^{\prime \prime \prime}<\sigma^{\prime \prime} / 2$ and for $r>\sigma^{\prime \prime} / 2$.

\section{References}

1. E. Bishop, Differentiable manifolds in complex Euclidean space, Duke Math. J. 32 (1965), 1-21.

2. F. ForstnerIČ, Analytic disks with boundaries in a maximal real submanifold of $\mathbb{C}^{2}$, Ann. Inst. Fourier 37 (1987), 1-44.

3. F. W. KamBER AND PH. TONDEUR, Characteristic invariants of foliated bundles, Manuscripta Mathematica 11 (1974), 51-89.

4. E. L. STOUT And W. Zame, Totally real imbeddings and the universal covering spaces of domains of holomorphy: some examples, Manuscripta Mathematica 50 (1985), 29-48.

5. R. O. WELLS, Compact real submanifolds of a complex manifold with nondegenerate holomorphic tangent bundles, Math. Ann. $\mathbf{1 7 9}$ (1969), 123-129.

6. M. Gromov, "Partial differential relations," Ergebnisse 3 folge Bd.9, Springer-Verlag, Berlin-Heidelberg-New York, 1986.

Tom Duchamp:

Department of Mathematics

University of Washington GN-50

Scattle, WA 98195

U.S.A.
Franc Forstnerič:

Department of Mathematics

University of Wisconsin

Madison, WI 53706

U.S.A. 